\title{
Eccentric endurance training in subjects with coronary artery disease: a novel exercise paradigm in cardiac rehabilitation?
}

Accepted: 17 September 2003/Published online: 27 November 2003 (C) Springer-Verlag 2003

\begin{abstract}
This study evaluated the effects of 8 weeks of eccentric endurance training (EET) in male subjects (age range 42-66 years) with coronary artery disease (CAD). EET was compared to concentric endurance training (CET) carried out at the same metabolic exercise intensity, three times per week for half an hour. CET $(n=6)$ was done on a conventional cycle ergometer and EET $(n=6)$ on a custom-built motordriven ergometer. During the first 5 weeks of the training program the metabolic load was progressively increased to $60 \%$ of peak oxygen uptake in both groups. At this metabolic load, mechanical work rate achieved was 97 (8) W [mean (SE)] for CET and 338 (34) W for EET, respectively. Leg muscle mass was determined by dual-energy X-ray absorptiometry, quadriceps strength with an isokinetic dynamometer and muscle fibre composition of the vastus lateralis muscle with morphometry. The leg muscle mass increased significantly in both groups by some $3 \%$. Strength parameters of knee extensors improved in EET only. Significant changes of $+11(4.9) \%,+15$ (3.2) $\%$ and $+9(2.5) \%$ were reached for peak isometric torque and peak concentric torques at $60^{\circ} \mathrm{s}^{-1}$ and $120^{\circ} \mathrm{s}^{-1}$, respectively. Fibre size increased significantly by $19 \%$ in CET only. In conclusion, the present investigation showed that EET is feasible in middleaged CAD patients and has functional advantages over
\end{abstract}

R. Steiner · K. Meyer · J.-P. Schmid · H. Saner Cardiovascular Prevention and Rehabilitation, Department of Cardiology, University Hospital, 3010 Bern, Switzerland

K. Lippuner

Osteoporosis Unit, University Hospital,

3010 Bern, Switzerland

H. Hoppeler $(\square)$

Department of Anatomy, University of Bern,

Bühlstrasse 26, 3009 Bern, Switzerland

E-mail: hoppeler@ana.unibe.ch

Tel.: + 41-31-6314637

Fax: + 41-31-6313807
CET by increasing muscle strength. Muscle mass increased similarly in both groups whereas muscle structural composition was differently affected by the respective training protocols. Potential limitations of this study are the cautiously chosen conditioning protocol and the restricted number of subjects.

Keywords Metabolic load - Work rate Strength Muscle mass $\cdot$ Muscle ultrastructure

\section{Introduction}

There is abundant evidence that physical activity delays the progression of coronary artery disease (CAD) by multiple biological mechanisms and may even lead to its regression (Shephard and Balady 1999; Fletcher et al. 2001). Traditionally, moderately intense aerobic exercise is known to mediate these health benefits (Pate et al. 1995) and has thus become a cornerstone in cardiac rehabilitation programs (Shephard and Balady 1999; Fletcher et al. 2001). Additionally, the importance of muscle strength has received attention in order to maintain a functional autonomy in the elderly (Frontera et al. 1988; Shephard and Balady 1999; Fletcher et al. 2001). In healthy subjects, muscle strength is classically trained with resistance training programs. Until recently, patients with cardiac diseases were discouraged from strength training because of the increase in cardiac afterload that is thought to occur during weightlifting exercises, particularly when strength training is carried out with resistances close to individual maximal loads (MacDougall et al. 1992; Shephard and Balady 1999). But it has been shown that there are no adverse effects of strength training in CAD when resistance exercise is carried out with a high number of repetitions and only moderate loads (Kelemen et al. 1986). Furthermore, an additional benefit for aerobic performance capacity, estimated as time to exhaustion, has been shown for this type of activity under certain conditions (Kelemen et al. 1986; McCartney et al. 1991). 
Eccentric muscle work is an essential part of our daily activities (Dickinson et al. 2000; Lindstedt et al. 2001). We use eccentric muscle work during normal walking, and in particular when walking downhill, to convert potential energy into heat. Eccentric muscle action is characterised by a low metabolic demand for a high power output. This was convincingly demonstrated in classical experiments involving concentric and simultaneous eccentric cycling on mechanically connected ergometers by Abbott et al. (1952). When carried out by unaccustomed subjects, eccentric muscle work can lead to substantial structural muscle damage followed by delayed onset muscle soreness (Fridén et al. 1983a). Repetitive bouts of eccentric work lead to adaptations making muscle more resistant to eccentric stress (Clarkson and Tremblay 1988). With chronic eccentric training muscle becomes essentially refractory to damage induced by eccentric contractions (Fridén et al. 1983b; LaStayo et al. 1999, 2000).

For development of muscle strength and to achieve muscle hypertrophy, combined concentric and eccentric muscle activation is recommended for resistance training programs at all fitness levels, from novice to advanced (Kraemer et al. 2002). Eccentric resistance training (Komi and Buskirk 1972; Colliander and Tesch 1990; Hortobágyi et al. 1996) and also eccentric endurance training (EET) on motor-driven ergometers (Fridén et al. 1983b; LaStayo et al. 2000) have been shown to improve muscle strength more than concentric training alone. Despite the high mechanical load on muscle tissue in eccentric exercise, the blood pressure response is similar to that of concentric exercise, provided that Valsalva manoeuvres are avoided (MacDougall et al. 1992). We reasoned that because of the high mechanical load that can be achieved during eccentric exercise this mode of training could be an option to achieve gains in muscular strength and eventually muscle mass at low cardiovascular demands.

We have previously shown that EET results in a similar haemodynamic response to concentric endurance training (CET) when carried out at the same metabolic workload. This, despite a several-fold higher mechanical work rate with EET than with CET. From this it was concluded that EET can be considered to be a safe training paradigm in this patient population (Meyer et al. 2003). The aim of the present study was to explore the structural and functional adaptation of EET in these CAD patients. Specifically, we hypothesised that EET provides a larger gain in muscle strength, muscle mass and muscle fibre size than CET when carried out at the same metabolic demand.

\section{Methods}

\section{Subjects and conditioning program}

Thirteen male patients with documented stable CAD who were participating in the outpatient cardiac rehabilitation program at University Hospital Bern were included in the study. The study protocol was approved by the local Ethics Committee on Human Research. Patients were informed about the study at the entry of the rehabilitation program and were clinically examined. After having given written informed consent they were randomly assigned to the CET or EET group. The randomisation procedure on the small number of subjects led to a considerable variability of some of the baseline characteristics. One patient in the EET group did not complete the entire study period. As a consequence there are some minor differences for the population means reported in a previous study on the same patient groups (Meyer et al. 2003). The anthropometrical characteristics of the remaining 12 subjects, as well as the medication they received, are presented in Table 1. Medication was not changed over the study period.

All tests reported below were performed before and repeated after the 8-week training period. EET or CET was integrated into a standard comprehensive rehabilitation program such that light callisthenics, stretching and relaxation procedures were identical for all subjects. Aerobic training was carried out by the CET group on an Ergometrics standard cycle ergometer (Ergoline, Bitz, Germany) whereas the EET group trained on a custom-built motordriven eccentric ergometer (Meyer et al. 2003). The entire training period lasted 8 weeks; CET and EET were carried out three times per week for half an hour. Training intensity was increased gradually over the first 5 weeks of the rehabilitation program to a level corresponding to $60 \%$ of the peak oxygen uptake $\left(\dot{V} \mathrm{O}_{2 \text { peak }}\right)$ achieved in the ramp test performed at the entry into the rehabilitation program. At this time invasive determination of central haemodynamic variables was carried out (Meyer et al. 2003). Because of ethical considerations we were not allowed to increase work rate after the catheter measurements. Therefore, exercise intensity was kept constant for the last 3 weeks of the program. At all times during the entire rehabilitation program we strived to have the CET and EET groups work at the same relative metabolic work intensity, as judged from their individual heart rates.

\section{Rating of perceived exertion}

Subjects were asked to report a rating of perceived exertion (RPE) every 5 min during each training session by using the Borg RPE-scale (6-20 rating) (Borg 1982). Separate ratings of the

Table 1 Baseline characteristics of patients by training groups. Values are means (SE), except disease categories* and medications, where the values are frequency counts. $B M I$ Body mass index, $\dot{V} \mathrm{O}_{2 \text { peak }}$ peak oxygen uptake, $M I$ myocardial infarction, $C A B G$ coronary artery bypass grafting, $P T C A$ percutaneous coronary angioplasty, $L V E F$ left ventricular ejection fraction, $A S A$ acetylsalicylic acid, $A C E$ angiotensin-converting enzyme, $A T_{1}$ angioten$\sin$ II type 1 receptor

\begin{tabular}{lll}
\hline & $\begin{array}{l}\text { Concentric } \\
(n=6)\end{array}$ & $\begin{array}{l}\text { Eccentric } \\
(n=6)\end{array}$ \\
\hline Age (years) & $56(3.5)$ & $55(2.6)$ \\
Body height $(\mathrm{cm})$ & $169(0.8)$ & $173(3.9)$ \\
Body mass $(\mathrm{kg})$ & $77(5.7)$ & $83(5.1)$ \\
BMI $\left(\mathrm{kg} \mathrm{m}^{-2}\right)$ & $27.1(1.9)$ & $27.6(1.3)$ \\
Body fat $(\%)$ & $25.4(2.9)$ & $21.1(2.1)$ \\
$\dot{V} \mathrm{O}_{2 \text { peak }}\left(\mathrm{ml} \mathrm{kg}^{-1} \mathrm{~min}^{-1}\right)$ & $28.0(2.6)$ & $28.7(2.1)$ \\
$\mathrm{MI}^{*}$ & 5 & 5 \\
$\mathrm{CABG}^{*}$ & 2 & 1 \\
$\mathrm{PTCA}^{*}$ & 3 & 5 \\
LVEF $(\%)$ & $65(3)$ & $56(3)$ \\
Medications & & 6 \\
$\quad$ ASA & 6 & 4 \\
Beta blockers & 4 & 3 \\
ACE inhibitors & 3 & 0 \\
AT ${ }_{1}$ antagonists & 1 & 1 \\
Ca channel blockers & 1 & 5 \\
Statins & 5 & 0 \\
Nitrates & 1 & 0 \\
Diuretics & 1 & \\
\hline
\end{tabular}


cardiorespiratory effort (dyspnoea) and of the specific leg exertion were requested.

Anthropometry and body composition

Body mass and height were obtained during the clinical examination. Body mass index (BMI) was calculated by dividing mass by height squared. Body composition was determined by fan-beam dual X-ray absorptiometry (DXA) on a QDR-4500A (Hologic Inc., Bedford, USA) before and after the training period. The DXA device measured lean mass, fat mass and bone mineral content of the whole body. Additionally, lower limbs were separated from trunk analysis by the means of the integrated software. Lower limb muscle mass was calculated from the measured fat-free mass according to an equation given by Shih et al. (2000). Values of the left and right limb were averaged for analysis.

\section{Muscle strength}

Muscle strength of the knee extensors was measured as peak torque on an isokinetic Cybex Norm dynamometer (Lumex, Ronkonkoma, USA). Before each test, subjects were familiarised with the testing device and the test procedures. Isometric peak torque was measured at a knee angle of $60^{\circ}$. Dynamic measurements were performed over a range of motion from $15^{\circ}$ to $100^{\circ}$ knee flexion (Kraemer and Fry 1995). Isokinetic concentric peak torque was measured at angular velocities of $+60^{\circ} \mathrm{s}^{-1}$ and $+120^{\circ} \mathrm{s}^{-1}$, isokinetic eccentric peak torque was measured at negative angular velocities of $-20^{\circ} \mathrm{s}^{-1}$ and $-60^{\circ} \mathrm{s}^{-1}$. Values of the left and right limb were averaged for analysis.

Muscle biopsies and morphometric analysis

Biopsies were to be taken before and after the training period, so heavy exercise was avoided $48 \mathrm{~h}$ prior to the biopsies. A sample of the right vastus lateralis muscle at mid-thigh level was obtained under local anaesthesia using the Bergström needle (Bergström 1962). Each sample was divided into two parts; one half was immediately frozen in isopentane-cooled liquid nitrogen and stored for later analysis. The other half was fixed in buffered glutaraldehyde for electron microscopy (Hoppeler et al. 1985). The ultrastructural analysis of the muscle fibres was performed using standard stereological methods (Weibel 1979). Ultra-thin transverse or slightly oblique sections were analysed by electron microscopy for capillary number and fibre cross-sectional area at a final magnification of approximately x 1,400 . The volume density of mitochondria, myofibrils and lipid droplets were estimated at a final magnification of approximately x 24,000 .

Choice of subjects and statistics

Because eccentric exercise training is not an established rehabilitation procedure in CAD patients we had to comply with requirements of the Ethical Committee which resulted in a difficulty of recruiting subjects for this study. Despite the restricted number of subjects, simple statistical analysis was performed. Two-tailed paired and unpaired Student's $t$-tests were applied to analyse intragroup changes from pre- to post-training and intergroup differences, respectively. The level of statistical significance was set at $P<0.05$.

\section{Results}

Over the entire training period we did not observe any complications in the cardiovascular or musculoskeletal systems. The final mechanical work rate achieved was close to $60 \%$ of $V \dot{V} \mathrm{O}_{2 \text { peak }}$, corresponding to $76 \%$ of the individual maximal heart rate, and was 97 (8) W for CET and 338 (34) W for EET, respectively $(P<0.001)$.

\section{Perceived exertion}

In none of the 8 weeks of training did the average RPE score during the sessions exceed 11 ('fairly light') in either the CET or the EET group. There was a tendency in CET to perceive cardiorespiratory effort slightly higher than leg effort whereas in the EET group the leg rating was generally higher, particularly during the first 5 weeks when exercise intensity was ramped up.

\section{Body composition and leg muscle mass}

BMI did not change over the training period in either of the groups. DXA showed an unchanged body fat content for EET [21.1 (2.1)\% before versus $21.6(1.7) \%$ after training], while a small but statistically significant decrease was noted in CET [25.4 (2.9)\% to 24.4 (2.9)\%; $P<0.001]$. Leg muscle mass increased significantly in both groups, with a similar magnitude. The gains were 191 (37) g per leg $(+2.7 \% ; P<0.01)$ for CET and 259 (93) g per leg $(+3.6 \% ; P<0.05)$ for EET, respectively. The increase between the groups was not statistically different.

\section{Muscle strength}

Changes in muscle strength for different testing modes are compiled in Fig. 1a for CET and Fig. 1b for EET.

For the CET group, peak isometric and peak isokinetic torques at all measured velocities did not change between pre- and post-training tests. In the EET group, isometric peak torque $[+11(4.9) \% ; P<0.05]$ and isokinetic concentric peak torque at slower $[+15(3.2) \%$; $P<0.01]$ and faster velocity $[+9(2.5) \% ; P<0.05]$ was increased, whereas the average increase of isokinetic eccentric peak torque for both velocities $(+6 \%)$ was not statistically significant.

\section{Muscle morphometry}

Muscle morphological characteristics are reported in Table 2. Qualitatively, normal muscle ultrastructure was preserved after training in both groups and no morphological abnormalities were observed during morphometric analysis of the tissue.

Muscle fibre cross-sectional area increased only in CET $(+19 \% ; P<0.05)$. Capillary supply, expressed as capillaries per muscle fibre, remained unchanged in both groups. The volume density of total mitochondria was 

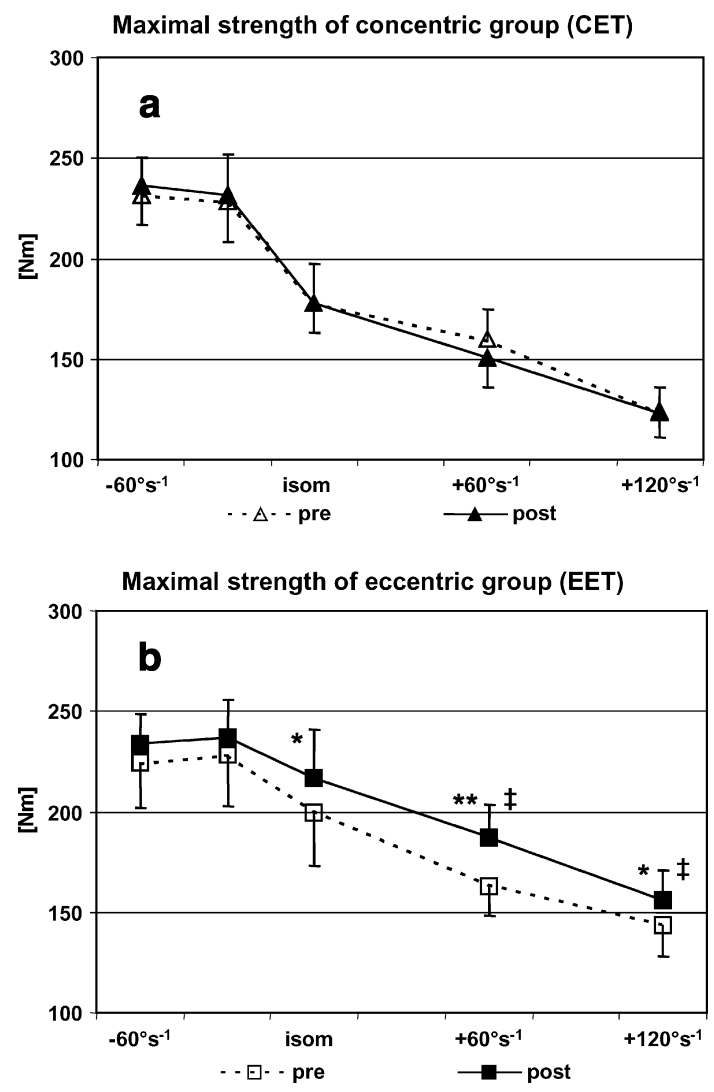

Fig. 1 a Maximal voluntary isokinetic peak torque of knee extensor muscles at different angular velocities before (pre) and after (post) 8 weeks of training on a concentric cycle ergometer. Values are means (SE). No statistically significant differences from pre- to post-test $(P=\mathrm{ns})$. b Maximal voluntary isokinetic peak torque of knee extensor muscles at different angular velocities before (pre) and after (post) 8 weeks of training on an eccentric cycle ergometer. Values are means (SE). Significant $\left({ }^{*} P<0.05\right.$, $* * P<0.01)$ difference from pre-test; ${ }^{\dagger}$ significantly $(P<0.05)$ more change than in the concentric group

also unchanged in both groups, despite an increased volume density of subsarcolemmal mitochondria in CET and a decrease of subsarcolemmal as well as interfibrillar mitochondria in the EET group. These mitochondrial changes are reflected in opposite changes of the myofibrils, which together with the remaining sarcoplasmic components (sarcoplasm, sarcoplasmic reticulum, lipid droplets and nuclei) make up the reference space, the muscle fibre volume. The volume density of lipids (approximately $0.5 \%$ of muscle fibre volume; not reported separately) was not significantly affected by either of the training interventions.

\section{Discussion}

The main finding of this study is a functional gain, i.e. improved muscular strength with EET compared to CET. However, this functional gain is not the consequence of the expected structural modifications. Leg muscle mass increased similarly in both groups, while muscle fibre size remained unchanged in EET. We therefore have to assume that this functional improvement is likely due to neuronal coordinative factors. As a limitation of this study we see the short period of 5 weeks available for ramping up the training intensity and thus the relatively low and constant workload that the subjects experienced over the last 3 weeks of training intervention. These constraints were required by ethical considerations of using a novel training paradigm for the first time in a CAD patient population.

Most studies on eccentric muscle work used few contractions at sub-maximal to maximal loads on isokinetic dynamometers (Colliander and Tesch 1990; Higbie et al. 1996; Hortobágyi et al. 1996). These studies therefore belong to the literature dealing with classical strength-type training paradigms. There are only a few studies with conditioning protocols comparable to the present study whereby eccentric load is applied continuously over the cranks of a cycle ergometer for time periods of $30 \mathrm{~min}$ per training session (Fridén et al. 1983b; LaStayo et al. 1999, 2000). However, these studies were realised with young healthy subjects and not with patients. To our knowledge EET has not previously been used as a part of a cardiac rehabilitation program. An important purpose of the present investigation, therefore, was to examine the feasibility of EET in middle-aged patients with $\mathrm{CAD}$ and to compare its effects on muscle mass, strength and muscle fibre

Table 2 Muscle structure before and after 8 weeks of concentric (CET, $n=6$ ) and eccentric (EET, $n=6$ ) cycle ergometer training, respectively. Values are means (SE). Mitochondria (central, subsarcolemmal, total), myofibrils and remaining components (incl. lipid droplets) are measured and expressed as percentage of volume

\begin{tabular}{|c|c|c|c|c|c|c|c|}
\hline & \multirow{2}{*}{$\begin{array}{l}\text { Fibre area } \\
\left(\mu \mathrm{m}^{2}\right)\end{array}$} & \multirow{2}{*}{$\begin{array}{l}\text { Capillaries } \\
\text { per fibre }(n)\end{array}$} & \multicolumn{3}{|c|}{ Mitochondria (\%) } & \multirow{2}{*}{$\begin{array}{l}\text { Myofibrils } \\
(\%)\end{array}$} & \multirow{2}{*}{$\begin{array}{l}\text { Remaining } \\
\text { components } \\
(\%)\end{array}$} \\
\hline & & & $\begin{array}{l}\text { Central } \\
(\%)\end{array}$ & $\begin{array}{l}\text { Subsarcolemmal } \\
(\%)\end{array}$ & $\begin{array}{l}\text { Total } \\
(\%)\end{array}$ & & \\
\hline \multicolumn{8}{|l|}{ CET } \\
\hline Pre & $3524(216)$ & $1.87(0.2)$ & $4.41(0.5)$ & $0.82(0.2)$ & $5.23(0.7)$ & $81.3(1.0)$ & $13.5(0.7)$ \\
\hline $\begin{array}{l}\text { Post } \\
\text { EET }\end{array}$ & $4209(417)^{*}$ & $2.04(0.2)$ & $4.64(0.4)$ & $1.16(0.2)^{*}$ & $5.97(0.5)$ & $77.5(0.9) *$ & $16.5(0.7)^{*}$ \\
\hline Pre & $4523(460)$ & $2.14(0.2)$ & $4.39(0.5)$ & $0.89(0.2)$ & $5.27(0.6)$ & $79.6(1.1)$ & $15.1(0.9)$ \\
\hline Post & $4402(282)$ & $2.07(0.2)$ & $3.67(0.2)$ & $0.53(0.1)$ & $4.20(0.3)$ & $81.8(0.7) *$ & $14.1(0.7)^{*}$ \\
\hline
\end{tabular}

*Intragroup difference $P<0.05$ 
ultrastructure to those obtained with concentric exercise carried out for the same time and at the same metabolic load. We could demonstrate that EET is feasible and well tolerated, as previously shown in younger healthy subjects (Fridén et al. 1983b; LaStayo et al. 1999, 2000). Despite a work rate almost 3.5-times higher in EET than in CET the RPEs were similarly low in both populations. The tendency for a slightly greater perceived effort of the legs with EET, observed in particular during ramping up the intensity (LaStayo et al. 1999), has previously been observed (Fridén et al. 1983b; LaStayo et al. 2000). The perception of muscle soreness was queried qualitatively. Some patients reported discreet muscle soreness for a maximum of $24 \mathrm{~h}$ after ramping. However, none of the trainings had to be cancelled due to muscle soreness.

We found no measurable effect of our training programs on BMI. With regard to the moderate exercise intensity in both groups, the short conditioning period and the absence of a nutritional intervention, this result is not surprising and similar to other exercise interventions of similar duration (Kelemen et al. 1986; Colliander and Tesch 1990). We consider the small change in body composition revealed by DXA in CET to be of negligible clinical relevance.

There is currently no data available for the influence of EET on leg muscle mass. We found a similar increase of leg muscle mass in both groups. It has been shown that concentric exercise training, even when carried out at higher workloads, does not lead to an increase in muscle cross-sectional area in previously untrained male subjects (Hoppeler et al. 1985). The fact that leg muscle mass is increased in both populations may be related to the relative state of deconditioning as a consequence of the primary pathology of our subjects. When eccentric exercise is used in a resistance training setting, muscle mass increases similarly (Jones and Rutherford 1987; Colliander and Tesch 1990) or more (Higbie et al. 1996) than when concentric resistance training is carried out. An additional consideration with regard to muscle hypertrophy is related to the medication taking by our patients. In animal experiments $\beta$-adrenergic receptor, i.e. the $B 2$-receptor, plays an important role for skeletal muscle hypertrophy (Hinkle et al. 2002). The importance of angiotensin II in acting on the type $1\left(\mathrm{AT}_{1}\right)$ receptor for muscle growth is judged ambiguously. In animal experiments angiotensin-converting enzyme inhibitors and $\mathrm{AT}_{1}$ antagonists both inhibit training-induced muscle hypertrophy (Gordon et al. 2001). On the other hand, in animal experiments angiotensin II can induce skeletal muscle wasting by enhancing protein degradation (Brink et al. 2001). Whether the medical treatment of our patients has influenced muscle hypertrophy negatively cannot be assessed from this study. In any case the patients in the EET and the CET group received nearly the same medication and must thus have been affected similarly.

By evaluating muscle strength with an isokinetic dynamometer, we found an average increase of $12 \%$ in peak isometric and peak isokinetic concentric torque with EET. This is similar to isokinetic values reported for resistance conditioning programs (Jones and Rutherford 1987; Frontera et al. 1988). Surprisingly, peak isokinetic eccentric torques increased only by $6 \%$. Due to training specificity we would have expected eccentric torques to improve more than concentric torques after an eccentric training regime (Higbie et al. 1996; Hortobágyi et al. 1996). However, it proved difficult to implement isokinetic eccentric torque measurements in this population. Clearly, the difficulties in coordinating eccentric movement lead to a greater variability of this testing procedure. Similar problems with estimating eccentric torques have previously been reported (Westing et al. 1988). Comparing the results in strength gain to those of studies with comparable conditioning protocols, we found them to be of similar magnitude to those reported by Fridén et al. (1983b), but only a third of those reported by LaStayo et al. (2000). We consider the main reason for this discrepancy to be the absolute work rate achieved at the end of the intervention period. Whereas the final work rate in the protocol used by LaStayo et al. (2000) was $489 \mathrm{~W}$, Fridén et al. (1983b) finished at $300 \mathrm{~W}$ and our study ended at $338 \mathrm{~W}$. It could be hypothesised that the observed difference in muscle response might be related to a mechanical threshold. No increase of maximal muscle strength was seen in CET between the pre- and post-training isokinetic tests. In view of the relatively low mechanical load of $97 \mathrm{~W}$ during the concentric training this is not surprising (McCartney et al. 1991; LaStayo et al. 1999, 2000). The structural findings indicate that the functional strength gains after EET can most likely be attributed to central factors (see also Colliander and Tesch 1990; Hortobágyi et al. 1996). This aspect deserves to be studied specifically in the future.

Muscle fibre size was found to be increased significantly by $19 \%$ in CET and to be unchanged in EET. This was contrary to our hypothesis as we would have expected EET to increase fibre size more than CET. It has previously been shown by LaStayo et al. (2000) that eccentric exercise training of similar duration (but higher intensity) led to an increase of fibre size of no less than $52 \%$. In contrast, Fridén et al. (1983b) using eccentric exercise at similar intensities as in our study did not report significant gains in muscle fibre size. In this context we have to consider that fibre size determined by morphometry is a biased estimate and its estimation in biopsy material is fraught with difficulties. Small differences, such as those suggested by the significant gains in muscle mass in EET and CET may therefore be difficult to resolve in biopsies (Zumstein et al. 1983). Moreover, we do not know whether the training regime used in the present study would influence the total fibre number; this would impact on fibre size. Capillarity assessed by the capillary to fibre ratio (uninfluenced by shifts in fibre size) remained constant in both populations. Total mitochondrial volume did not change significantly in 
either of the populations; however, we found an increase in mitochondrial volume density of $14 \%$ in CET. This increase was mainly due to a significant $(42 \%)$ increase in the population of subsarcolemmal mitochondria. These findings taken together can be seen as an adaptation to the low intensity endurance type exercise carried out by CET (Hoppeler 1986). With regard to EET there is very little information available in the literature. LaStayo et al. (2000) found mitochondrial volume density to be unchanged in eccentric exercise training, albeit carried out at much higher workloads and more often per week, while Fridén et al. (1983b) found mitochondrial volume densities (obtained from longitudinal sections) to be increased. His data show surprisingly low initial mitochondrial densities, which may partly be responsible for the observed significant increases after EET. As it stands now it is too early to decide on the effect of EET on the mitochondrial populations in muscle fibres.

In conclusion, we have shown that eccentric exercise training is feasible in middle-aged men with CAD and without musculoskeletal complication. It provides functional improvements by increasing muscular strength, presumably as a result of the 3.5-times higher eccentric mechanical work rate achieved at a given metabolic load. The increase in muscle mass was found to be similar in concentric and eccentric exercise while muscle fibre size and ultrastructure were not significantly altered after EET. It will be necessary to reach higher workloads in eccentric training in future studies to further explore the potential of this novel training paradigm.

Acknowledgements We thank Christoph Lehmann for technical and Liliane Tüscher-Gfeller for clerical support. We thank also Sharzad Naderi and Daniel Troxler for the Cybex measurements, Franziska Graber for morphometric muscle analysis and Hans Howald for proofreading and checking consistency of the manuscript.

\section{References}

Abbott BC, Bigland B, Ritchie JM (1952) The physiological cost of negative work. J Physiol (Lond) 117:380-390

Bergström J (1962) Muscle electrolytes in man. Scand J Clin Lab Invest 14[Suppl 68]:11-13

Borg GA (1982) Psychophysical bases of perceived exertion. Med Sci Sports Exerc 14:377-381

Brink M, Price SR, Chrast J, Bailey JL, Anwar A, Mitch WE, Delafontaine P (2001) Angiotensin II induces skeletal muscle wasting through enhanced protein degradation and down-regulates autocrine insulin-like growth factor I. Endocrinology 142:1489-1496

Clarkson PM, Tremblay I (1988) Exercise-induced muscle damage, repair, and adaptation in humans. J Appl Physiol 65:1-6

Colliander EB, Tesch PA (1990) Effects of eccentric and concentric muscle actions in resistance training. Acta Physiol Scand 140:31-39

Dickinson MH, Farley CT, Full RJ, Koehl MA, Kram R, Lehman S (2000) How animals move: an integrative view. Science 288:100-106

Fletcher GF, Balady GJ, Amsterdam EA, Chaitman B, Eckel R, Fleg J, Froelicher VF, Leon AS, Pina IL, Rodney R, SimonsMorton DA, Williams MA, Bazzarre T (2001) Exercise stan- dards for testing and training: a statement for healthcare professionals from the American Heart Association. Circulation 104:1694-1740

Fridén J, Sjöström M, Ekblom B (1983a) Myofibrillar damage following intense eccentric exercise in man. Int $\mathbf{J}$ Sports Med 4:170-176

Fridén J, Seger J, Sjöström M, Ekblom B (1983b) Adaptive response in human skeletal muscle subjected to prolonged eccentric training. Int J Sports Med 4:177-183

Frontera WR, Meredith CN, O'Reilly KP, Knuttgen HG, Evans WJ (1988) Strength conditioning in older men: skeletal muscle hypertrophy and improved function. J Appl Physiol 64:10381044

Gordon SE, Davis BS, Carlson CJ, Booth FW (2001) ANG II is required for optimal overload-induced skeletal muscle hypertrophy. Am J Physiol Endocrinol Metab 280:E150-159

Higbie EJ, Cureton KJ, Warren GL 3rd, Prior BM (1996) Effects of concentric and eccentric training on muscle strength, crosssectional area, and neural activation. J Appl Physiol 81:21732181

Hinkle RT, Hodge KM, Cody DB, Sheldon RJ, Kobilka BK, Isfort RJ (2002) Skeletal muscle hypertrophy and anti-atrophy effects of clenbuterol are mediated by the beta2-adrenergic receptor. Muscle Nerve 25:729-734

Hoppeler H (1986) Exercise-induced ultrastructural changes in skeletal muscle. Int J Sports Med 7:187-204

Hoppeler H, Howald H, Conley K, Lindstedt SL, Claassen H, Vock P, Weibel ER (1985) Endurance training in humans: aerobic capacity and structure of skeletal muscle. J Appl Physiol 59:320-327

Hortobágyi T, Hill JP, Houmard JA, Fraser DD, Lambert NJ, Israel RG (1996) Adaptive responses to muscle lengthening and shortening in humans. J Appl Physiol 80:765-772

Jones DA, Rutherford OM (1987) Human muscle strength training: the effects of three different regimens and the nature of the resultant changes. J Physiol (Lond) 391:1-11

Kelemen MH, Stewart KJ, Gillilan RE, Ewart CK, Valenti SA, Manley JD, Kelemen MD (1986) Circuit weight training in cardiac patients. J Am Coll Cardiol 7:38-42

Komi PV, Buskirk ER (1972) Effect of eccentric and concentric muscle conditioning on tension and electrical activity of human muscle. Ergonomics 15:417-434

Kraemer WJ, Fry AC (1995) Strength testing: development and evaluation of methodology. In: Maud PJ, Foster C (eds) Physiological assessment of human fitness. Human Kinetics, Champaign, Ill., pp 115-138

Kraemer WJ, Adams K, Cafarelli E, Dudley GA, Dooly C, Feigenbaum MS, Fleck SJ, Franklin B, Fry AC, Hoffman JR, Newton RU, Potteiger J, Stone MH, Ratamess NA, TriplettMcBride T (2002) American College of Sports Medicine position stand. Progression models in resistance training for healthy adults. Med Sci Sports Exerc 34:364-380

LaStayo PC, Reich TE, Urquhart M, Hoppeler H, Lindstedt SL (1999) Chronic eccentric exer-cise: improvements in muscle strength can occur with little demand for oxygen. Am J Physiol 276:R611-615

LaStayo PC, Pierotti DJ, Pifer J, Hoppeler H, Lindstedt SL (2000) Eccentric ergometry: increases in locomotor muscle size and strength at low training intensities. Am J Physiol Regul Integr Comp Physiol 278:R1282-1288

Lindstedt SL, LaStayo PC, Reich TE (2001) When active muscles lengthen: properties and consequences of eccentric contractions. News Physiol Sci 16:256-261

MacDougall JD, McKelvie RS, Moroz DE, Sale DG, McCartney N, Buick F (1992) Factors affecting blood pressure during heavy weight lifting and static contractions. J Appl Physiol 73:1590-1597

McCartney N, McKelvie RS, Haslam DR, Jones NL (1991) Usefulness of weightlifting training in improving strength and maximal power output in coronary artery disease. Am J Cardiol 67:939-945 
Meyer K, Steiner R, LaStayo PC, Lippuner K, Allemann Y, Eberli FR, Schmid J-P, Saner H, Hoppeler H (2003) Eccentric exercise in coronary patients: central hemodynamic and metabolic responses. Med Sci Sports Exerc 35:1076-1082

Pate RR, Pratt M, Blair SN, Haskell WL, Macera CA, Bouchard C, Buchner D, Ettinger W, Heath GW, King AC, et al (1995) Physical activity and public health. A recommendation from the Centers for Disease Control and Prevention and the American College of Sports Medicine. Jama 273:402-407

Shephard RJ, Balady GJ (1999) Exercise as cardiovascular therapy. Circulation 99:963-972

Shih R, Wang Z, Heo M, Wang W, Heymsfield SB (2000) Lower limb skeletal muscle mass: development of dual-energy
X-ray absorptiometry prediction model. J Appl Physiol 89:1380-1386

Weibel ER (1979) Stereological Methods, vol I. Academic Press, London

Westing SH, Seger JY, Karlson E, Ekblom B (1988) Eccentric and concentric torque-velocity characteristics of the quadriceps femoris in man. Eur J Appl Physiol 58:100-104

Zumstein A, Mathieu O, Howald H, Hoppeler H (1983) Morphometric analysis of the capillary supply in skeletal muscles of trained and untrained subjects: its limitations in muscle biopsies. Pflugers Arch 397:277-283 